BMJ Open Diabetes

Research $\&$ Care

\title{
Comparison of the effects of lockdown due to COVID-19 on glucose patterns among children, adolescents, and adults with type 1 diabetes: CGM study
}

\author{
Guido Di Dalmazi (D) , ', Giulio Maltoni, ${ }^{2}$ Claudio Bongiorno (D) , ${ }^{1}$ Lorenzo Tucci, ${ }^{1}$ \\ Valeria Di Natale, ${ }^{2}$ Simona Moscatiello, ${ }^{1}$ Gilberto Laffi, ${ }^{1}$ Andrea Pession, ${ }^{2}$ \\ Stefano Zucchini, ${ }^{2}$ Uberto Pagotto ${ }^{1}$
}

To cite: Di Dalmazi G, Maltoni G, Bongiorno C, et al. Comparison of the effects of lockdown due to COVID-19 on glucose patterns among children, adolescents, and adults with type 1 diabetes: CGM study. BMJ Open Diab Res Care 2020;8:e001664. doi:10.1136/ bmjdrc-2020-001664

- Supplemental material is published online only. To view, please visit the journal online (http://dx.doi.org/10.1136/ bmjdrc-2020-001664).

Received 22 June 2020 Revised 14 September 2020 Accepted 21 September 2020

D Check for updates

(C) Author(s) (or their employer(s)) 2020. Re-use permitted under CC BY-NC. No commercial re-use. See rights and permissions. Published by BMJ.

${ }^{1}$ Endocrinology and Diabetes Prevention and Care Unit, Department of Medical and Surgical Sciences, University of Bologna, Bologna, Italy ${ }^{2}$ Unit of Pediatrics, Department of Medical and Surgical Sciences, University of Bologna, Bologna, Italy

Correspondence to Professor Uberto Pagotto; uberto.pagotto@unibo.it

\section{ABSTRACT}

Introduction The COVID-19 pandemic forced the Italian government to issue extremely restrictive measures on daily activities since 11 March 2020 ('lockdown'), which may have influenced the metabolic control of type 1 diabetes mellitus (T1D). The aims of the study were to investigate continuous glucose monitoring (CGM) metrics in children and adults with T1D during lockdown and to identify their potentially related factors.

Research design and methods We enrolled 130 consecutive patients with T1D ( 30 children ( $\leq 12$ years), 24 teenagers (13-17 years), and 76 adults ( $\geq 18$ years)) using either Dexcom or FreeStyle LibreCGM $>70 \%$ during the study period, without hybrid closed-loop insulin pump. CGM metrics during the 20 days before and the 20 days after lockdown were calculated. By telephonic contact, we performed validated physical activity and perceived stress questionnaires.

Results In children, significantly lower glucose SD (SDglu) $(p=0.029)$ and time below range $(T B R)<54 \mathrm{mg} /$ $\mathrm{dL}$ (TBR2) ( $p=0.029)$ were detected after lockdown. CGM metrics were comparable in teenagers before and during lockdown. After lockdown, adults improved significantly time in range $(\mathrm{TIR}) 70-180 \mathrm{mg} / \mathrm{dL}(\mathrm{p}<0.001)$ and remaining metrics, except percent coefficient of variation and TBR2. In adults, considering the changes in SDglu and TIR occurred before and during lockdown, we identified a group with improved TIR and SDglu who performed more physical activity, one with improved glucose variability who was younger than the other patients, and one with worsened glucose variability who showed higher perceived stress than others.

Conclusion In patients with T1D during lockdown, CGM metrics mostly improved in children and adults, whereas it was unchanged in teenagers. In adults, age, physical activity, and perceived stress may be relevant contributing factors.

\section{INTRODUCTION}

After the first outbreak in China, Italy was one of the first countries worldwide that was significantly affected by the SARS-CoV-2 pandemic. ${ }^{2}$ The Italian government was forced to apply strong measures to prevent the

\section{Significance of this study}

What is already known about this subject?

- Time in range and SD of glucose measured by CGM improved in adult patients with type 1 diabetes during lockdown due to the COVID-19 pandemic.

What are the new findings?

- Continuous glucose monitoring (CGM) parameters during lockdown improved in children, whereas remained unchanged in teenagers.

- Despite adults improved their CGM parameters during lockdown, $25 \%$ worsened their glucose variability due to high perceived stress.

How might these results change the focus of research or clinical practice?

- This study highlights the different impact of a more regular daily routine in children and teenagers with type 1 diabetes. Moreover, the study provides a stratification of adult patients, giving information of categories of patients at risk of worsening their glucose variability during lockdown, and their associated factors. Those results indeed may be extremely useful in identifying adult patients who deserve a closer attention in the case of a new lockdown.

spread of the COVID-19 infection, by issuing extremely restrictive measures on people's daily activities and movements across the Country (hereby called 'lockdown'). Indeed, starting from 11 March 2020, non-essential businesses were closed, and outdoors sports, leisure activities, and travels between different cities have been severely limited. ${ }^{3}$ Prior to that date, during the third week of February 2020, schools and universities more cautiously suspended their educational services.

In this scenario, adult patients affected by type 1 diabetes mellitus (T1D) may be considered a vulnerable population, since hypertension and diabetes are known risk 
factors for severe COVID-19-related pneumonia. ${ }^{4-7}$ As the treatment mainstay of T1D is insulin administration, either via multiple daily injections (MDIs) or continuous subcutaneous insulin infusion devices, based on food intake, glucose concentrations, and physical activity, the fine-tuned balance between daily activities and therapeutic actions can be endangered by changes to the daily routine. Additionally, psychological stress may play a role in the worsening of glucose levels in patients with T1D contributing to the development of severe respiratory disease in the case of COVID-19 infection.

Continuous glucose monitoring (CGM) devices have improved the management of T1D by easing glucose monitoring, enabling an easier remote sharing of glucose data with healthcare providers, even in real time..$^{8-12}$ Therefore, the use of CGM devices could be relevant for the management of T1D during lockdown. A very recent study published during the writing of this manuscript has shown that glucose control improved after the lockdown in middle-aged adults with T1D under CGM. ${ }^{13}$ Two additional more recent studies published while the article was under review confirmed that adult patients did not worsen ${ }^{14}$ or improve ${ }^{15}$ their glucose profile during lockdown.

To date, no analysis of CGM data has been performed in children during lockdown. Furthermore, no extensive investigation has been performed to identify subgroups of patients who may have a more difficult glucose control during lockdown. The first aim of our study was to investigate the time in range (TIR) and the glucose variability as measured by CGM in children and adults with T1D during lockdown. The second aim was to identify the factors potentially contributing to the glycemic control under lockdown during the COVID-19 pandemic.

\section{METHODS}

Patients

We retrieved data from 188 consecutive patients wearing CGM system in active follow-up for T1D at the endocrinology and diabetes prevention and care unit and at the unit of pediatric diabetology of the S. Orsola Policlinic. Inclusion criteria were as follows: T1D with disease duration $>1$ year, age $\leq 70$ years, use of Dexcom or FreeStyle Libre monitoring systems, CGM with a sensor use of $>70 \%$ during the time range between 17 February 2020 and 5 April 2020, MDI therapy or non-automated insulin pump, and permission to access the CGM data remotely. Exclusion criteria were pregnancy, gestational diabetes, use of corticosteroid or drugs known to have a relevant impact on glycemic control during the 6 months before study period, use of hybrid closed loop insulin pump. After applying inclusion and exclusion criteria, we finally enrolled 130 patients, 30 children ( $\leq 12$ years), 24 teenagers (13-17 years), and 76 adults ( $\geq 18$ years). All patients were living in the area surrounding Bologna and were under the same constraint public rules.

\section{Study design}

We collected glucose data derived from CGM during the 20 days before the lockdown in Italy, from 20 February 2020 to 10 March 2020 (prelockdown) and 20 days starting from that date, from 11 to 30 March 2020 (during lockdown). Given that during the prelockdown period the schools in Italy were already closed and school-age patients had changed their routine activities already, we also collected data derived from CGM during an additional antecedent period of 20 days, from 30 January 2020 to 19 February 2020, while schools were still open (school period). In this specific time period, CGM data derived from sensor use of $>70 \%$ were available in $25 / 30$ children, 20/24 teenagers, and 71/76 adults.

We included patients using either Dexcom G5 or G6 (Dexcom San Diego, California) or FreeStyle Libre (Abbott Laboratories, Abbott Park, Illinois) systems. Raw glucose data were downloaded from the CGM systemspecific web service in the form of a comma-separated value spreadsheet for each patient. The CGM metrics described further were then calculated for each patient using a specifically designed application developed using the SciPy ecosystem, running on top of the Python V.3.7.6 programming language. We calculated mean and median glucose, glucose management indicator, glucose SD (SDglu), percent coefficient of variation (\%CV), TIR $70-180 \mathrm{mg} / \mathrm{dL}$, time in hypoglycemia $54-69 \mathrm{mg} /$ $\mathrm{dL}$ (time below range (TBR)-TBR1) and $<54 \mathrm{mg} / \mathrm{dL}$ (TBR2), time in hyperglycemia $180-250 \mathrm{mg} / \mathrm{dL}$ (time above range (TAR) - TAR1) and $>250 \mathrm{mg} / \mathrm{dL}$ (TAR2), and the risk indices High blood Glucose Index and Low Blood Glucose Index (LBGI) according to previous publications. ${ }^{16-21}$ In FreeStyle Libre users, we calculated the number of scans per day. We also recorded the most recent value of glycated hemoglobin (within 4 months before study period), duration of diabetes, type of insulin therapy, mean daily dose of insulin, and anthropometric measures at the last medical visit. SD score for body mass index was calculated in children according to a previous publication. ${ }^{22}$ We also recorded the date of last consultation and whether medical advice was performed during the lockdown period either in person visit or telephonic contact.

Patients and parents (in the case of minors) were contacted by phone within 2 weeks after the study period to collect data on COVID-19 infection or intercurrent communicable diseases and number of episodes of severe hypoglycemia. In adult patients, we also investigated whether they were working outside of the home during lockdown or if they had undergone smart working at home. Additionally, during the same telephonic contact, we administered the International Physical Activity Questionnaire-Short Form (IPAQ-SF) ${ }^{23}$ to all patients and the 10-Item Italian Perceived Stress Scale questionnaire ${ }^{24}$ (PSS) to adult patients. The IPAQ-SF, investigating physical activity, relies on nine items providing information on the time spent walking, in high-intensity activities, in 
activities of moderate intensity, and in sedentary activity. Data from the IPAQ-SF provide an estimate of the total weekly physical activity by weighting the reported minutes per week within each activity category by a metabolic equivalent (MET) energy expenditure estimate assigned to each category of activity, based on the 2000 compendium of physical activities (moderate-intensity activities had between 3 and 6 METs and vigorous-intensity activities had $>6$ METs). The time spent sitting per week was also estimated. The questionnaire Perceived Stress Scale assesses the degree to which aspects of the life are perceived as uncontrollable, unpredictable, and overloaded. Through investigating 10 items, the questionnaire evaluates thoughts and feelings of subjects referring to stressful events that occurred in the month before the detection.

\section{Statistical analysis}

Data are shown as median and interquartile range (IQR), as recommended by the international consensus, ${ }^{18}$ if not otherwise specified. Continuous variables were analyzed by one-way analysis of variance, and categorical variables were investigated by $\chi^{2}$ test. Significance values in multiple comparisons have been adjusted by the Bonferroni correction for multiple tests as a post hoc analysis. In the $\chi^{2}$ test performed in more than three groups, the column proportion test was used to investigate pairwise comparisons, after adjustment for Bonferroni correction as a post hoc analysis. Comparisons of paired data (school period-prelockdown and prelockdown-during lockdown) were performed by paired-samples t-test. The unsupervised clustering analysis was performed in adult patients to identify homogeneous groups of subjects based on specific parameters. Such a technique uses an algorithm that starts with each patient in a separate cluster and combines clusters until only one is left. We performed unsupervised clustering by using the following two parameters: percent difference between prelockdown period and during lockdown of TIR $(\Delta \mathrm{TIR})$ and SDglu ( $\Delta$ SDglu $)$, calculated as [(during lockdown value $\times 100) /$ prelockdown value]-100. For unsupervised clustering, we used Ward's method with squared Euclidean distance as a measure of interval. We finally selected three groups for subsequent analysis. We have chosen TIR as a parameter of adequacy of glycemic control and SDglu as a measure of glycemic variability. To perform unsupervised clustering, we did not consider \% CV among parameters of glycemic variability because it was not significantly different between the time periods before and during lockdown neither in the whole groups of patients nor in the three groups divided by age-range (children, teenagers, and adults), according to paired-samples comparison. Therefore, $\% \mathrm{CV}$ would not have led to a clear separation of groups following unsupervised clustering. Statistical analysis was performed by SPSS V.26. P values of $<0.05$ were considered significant.

\section{RESULTS}

Anthropometric and biochemical characteristics are shown in table 1 .

The number of adult patients using FreeStyle Libre was significantly higher than that of children and teenagers $(p<0.001)$. A significantly higher proportion of adult patients was under MDI therapy $(\mathrm{p}<0.001)$, with significantly lower doses of insulin than remaining groups $(p<0.001)$. Glycated hemoglobin at last visit was not significantly different among groups $(\mathrm{p}=0.080)$. According to the physical activity questionnaire results, moderate physical activity during lockdown was significantly lower in teenagers $(p=0.001)$, even though the global mean daily physical activity was not different among groups. Among school-age patients, the mean daily time spent sitting was significantly higher in teenagers than in children $(p=0.005)$. None but one adult patient had confirmed COVID-19 infection, which resolved without specific treatment and did not require hospital admission.

The comparison of CGM data before and after lockdown in the whole population showed that \%CV, TBR1, TBR2, and LBGI were comparable between the two time periods, whereas all remaining CGM-derived metrics were significantly lower, except TIR, which was significantly higher, after lockdown (online supplemental table 1). The number of scans per day in FreeStyle Libre users was similar between prelockdown (mean \pm SD 11.0 \pm 4.5$)$ and during lockdown $(11.2 \pm 4.8) \quad(\mathrm{p}=0.658)$. The paired analysis prelockdown-during lockdown performed by age classes is shown in table 2.

In children, SDglu, TBR2, and LBGI were significantly lower after lockdown, whereas no change in the remaining CGM metrics was recorded, when compared with the prelockdown period. Conversely, all CGM-derived metrics were comparable between prelockdown and during lockdown in teenagers. In adults, TIR was significantly higher $(\mathrm{p}<0.001)$, and all remaining parameters were significantly lower after lockdown, except for $\% \mathrm{CV}$ and TBR2, which were comparable between the time periods considered. A slight worsening of TBR1 was also observed $(\mathrm{p}=0.019)$. The mean insulin dose $/ \mathrm{kg}$ body weight/ day was slightly higher during lockdown than before lockdown $(0.55 \pm 0.18 \mathrm{U} / \mathrm{kg} /$ day vs $0.56 \pm 0.18 \mathrm{U} / \mathrm{kg} /$ day, $\mathrm{p}=0.015$ ). Considering the recently published data from the T1D exchange study, ${ }^{25}$ showing that glycemic control of people aged 18-25 are worse than children and older people ( $\geq 26$ years of age), we performed a separate analysis of CGM metrics in patients aged 18.0-25.9 years old $(\mathrm{n}=10)$. In this group of patients, prelockdown TIR was significantly lower than TIR during lockdown (median $65.9 \%$, IQR 44.7-76.1 vs median 68.9\%, IQR 53.3-78.5; $\mathrm{p}=0.027$ ), whereas SDglu was significantly lower during lockdown (prelockdown median 56.0\%, IQR 48.6-73.6 vs median during lockdown 49.8, IQR 46.0-71.2; $\mathrm{p}=0.005$ ). No significant differences could be detected in the remaining CGM metrics. 
Table 1 Anthropometric and biochemical characteristics at baseline

\begin{tabular}{|c|c|c|c|c|}
\hline & $\begin{array}{l}\text { Children } \\
\text { ( } \leq 12 \text { years) }\end{array}$ & $\begin{array}{l}\text { Teenagers } \\
\text { (13-17 years) }\end{array}$ & $\begin{array}{l}\text { Adults } \\
\text { ( } \geq 18 \text { years) }\end{array}$ & $P$ value \\
\hline Number of patients & 30 & 24 & 76 & \\
\hline Age (years) & $8.8(7.7-10.6)$ & $15.6(14.2-16.8)$ & $45.0(29.0-58.1)$ & $<0.001$ \\
\hline Female, n (\%) & $13(43.3)$ & $9(37.5)$ & $37(48.7)$ & 0.611 \\
\hline SDS-BMI or BMI* & $-0.2(-0.5$ to 0.4$)$ & $21.3(19.8-23.1)$ & $24.7(22.1-26.8)$ & n.a. \\
\hline Diabetes duration (years) & $4.2(2.3-6.5)$ & $7.2(5.1-9.5)$ & $22.0(14.3-30.8) \dagger$ & $<0.001$ \\
\hline \multicolumn{5}{|l|}{ Complications } \\
\hline Any, n (\%)‡ & $30(100.0)$ & $24(100.0)$ & $54(71.1)$ & \multirow[t]{5}{*}{0.016} \\
\hline Diabetic retinopathy, n (\%) & $0(0.0)$ & $0(0.0)$ & $12(15.8)$ & \\
\hline Diabetic neuropathy, n (\%) & $0(0.0)$ & $0(0.0)$ & $2(2.6)$ & \\
\hline Diabetic foot, n (\%) & $0(0.0)$ & $0(0.0)$ & $1(1.3)$ & \\
\hline >1 complication, $\mathrm{n}(\%)$ & $0(0.0)$ & $0(0.0)$ & $7(9.2)$ & \\
\hline Dexcom/FreeStyle Libre users, n (\%)§ & 28/2 (93.3/6.7) & 22/2 (91.7/8.3) & $35 / 41(46.1 / 53.9) \dagger$ & $<0.001$ \\
\hline Patients under multiple daily injection therapy, $\mathrm{n}(\%)$ & $12(40.0)$ & $11(45.8)$ & $63(82.9) \dagger$ & $<0.001$ \\
\hline Mean insulin dose (U/kg/day) & $0.8(0.6-0.8)$ & $0.8(0.7-1)$ & $0.5(0.4-0.6)$ & $<0.001$ \\
\hline Glycated hemoglobin, $\mathrm{mmol} / \mathrm{mol}$ & $57(51-62)$ & $51(46-57)$ & $56(49-64)$ & 0.080 \\
\hline \multicolumn{5}{|l|}{ During lockdown } \\
\hline Patients receiving medical advice by telephone, $\mathrm{n}(\%)$ & $6(20.0)$ & $2(8.0)$ & $8(11.0)$ & 0.330 \\
\hline Patients with severe hypoglycemia, n (\%) & $0(0.0)$ & $0(0.0)$ & $1(1.3)$ & n.a. \\
\hline IPAQ: total physical activity/day, METs & $1440(840-1920)$ & $1018(480-3056)$ & $1680(720-3180)$ & 0.524 \\
\hline IPAQ: high-intensity physical activity/day, METs & $0(0-320)$ & $180(0-2880)$ & $440(0-1560)$ & 0.104 \\
\hline IPAQ: moderate physical activity/day, METs & $1440(240-1680)$ & $100(0-390) \dagger$ & $480(120-1080)$ & 0.001 \\
\hline IPAQ: walking/day, METs & $0(0-180)$ & $75(0-428)$ & 350 (78-780)ף & 0.001 \\
\hline IPAQ: mean sitting time/day, hours & $4.0(3.0-6.0)$ & $7.3(5.0-8.6) \uparrow$ & $5.5(4-7.8)$ & 0.005 \\
\hline PSS: perceived stress & n.a. & n.a. & $14.5(9.8-20)$ & n.a. \\
\hline \multicolumn{5}{|l|}{ Patients employed in working activity } \\
\hline No, n (\%) & n.a. & n.a. & $36(47.4)$ & \multirow[t]{3}{*}{ n.a. } \\
\hline Yes, n (\%) & n.a. & n.a. & $20(26.3)$ & \\
\hline Smart working at home, $\mathrm{n}(\%)$ & n.a. & n.a. & $20(26.3)$ & \\
\hline
\end{tabular}

Data are expressed as median with IQR in parentheses. Continuous variables were analyzed by one-way analysis of variance, and categorical variables were investigated by $\chi^{2}$ test. Pairwise comparisons were made after Bonferroni correction.

${ }^{*}$ In children, SDS-BMI was calculated instead of BMI, according to a previous publication. ${ }^{21}$

$\dagger \mathrm{P}<0.05$ vs other groups.

$\ddagger$ No diabetic retinopathy, no diabetic neuropathy, and no diabetic foot.

$\S$ The number of scans per day in FreeStyle Libre users was similar between prelockdown (mean \pm SD $11.0 \pm 4.5)$ and during lockdown (11.2 \pm 4.8 ) $(p=0.658)$.

\P<0.05 vs children.

BMI, body mass index; IPAQ, International Physical Activity Questionnaire-Short Form; MET, metabolic equivalent; n.a., not assessed; PSS,

Perceived Stress Scale; SDS-BMI, SD score for body mass index.

The analysis of paired samples between the schoolopen period and the prelockdown period did not show significant differences between the two time periods for all parameters, except TAR1, which was slightly increased in children (25.5 (21.8-31.3) vs $28.1 \quad(25.1-31.0)$; $\mathrm{p}=0.047)$, and SDglu, which was lower in teenagers $(62.6$ (54.8-69.3) vs 59.1 (48.6-66.9), $\mathrm{p}=0.041$ ) (online supplemental table 2). No difference was detected among CGM metrics between the two time periods in adults.

The unsupervised clustering analysis performed in adults to stratify patients according to their change in TIR and SDglu as measure of glycemic variability before and after lockdown led to identification of three groups: a group with significantly higher $\Delta \mathrm{TIR}$ and lower $\Delta \mathrm{SDglu}$ than other groups, named improved TIR and variability' ( $\mathrm{n}=19,25 \%)$, and two groups with comparable $\Delta \mathrm{TIR}$ values but different $\Delta \mathrm{SD}$, which was decreased in one (named 'improved variability') $(\mathrm{n}=38,50 \%)$ and increased in the other (named 'worsened variability') $(\mathrm{n}=19,25 \%)$, respectively. As shown in table 3 , patients belonging to the group improved variability were significantly younger than the other groups $(\mathrm{p}=0.003)$ and had lower levels of glycated hemoglobin prior to lockdown than the improved TIR and variability group $(\mathrm{p}=0.031)$. Global mean daily physical activity measured by IPAQ-SF was significantly higher in the improved TIR and variability 


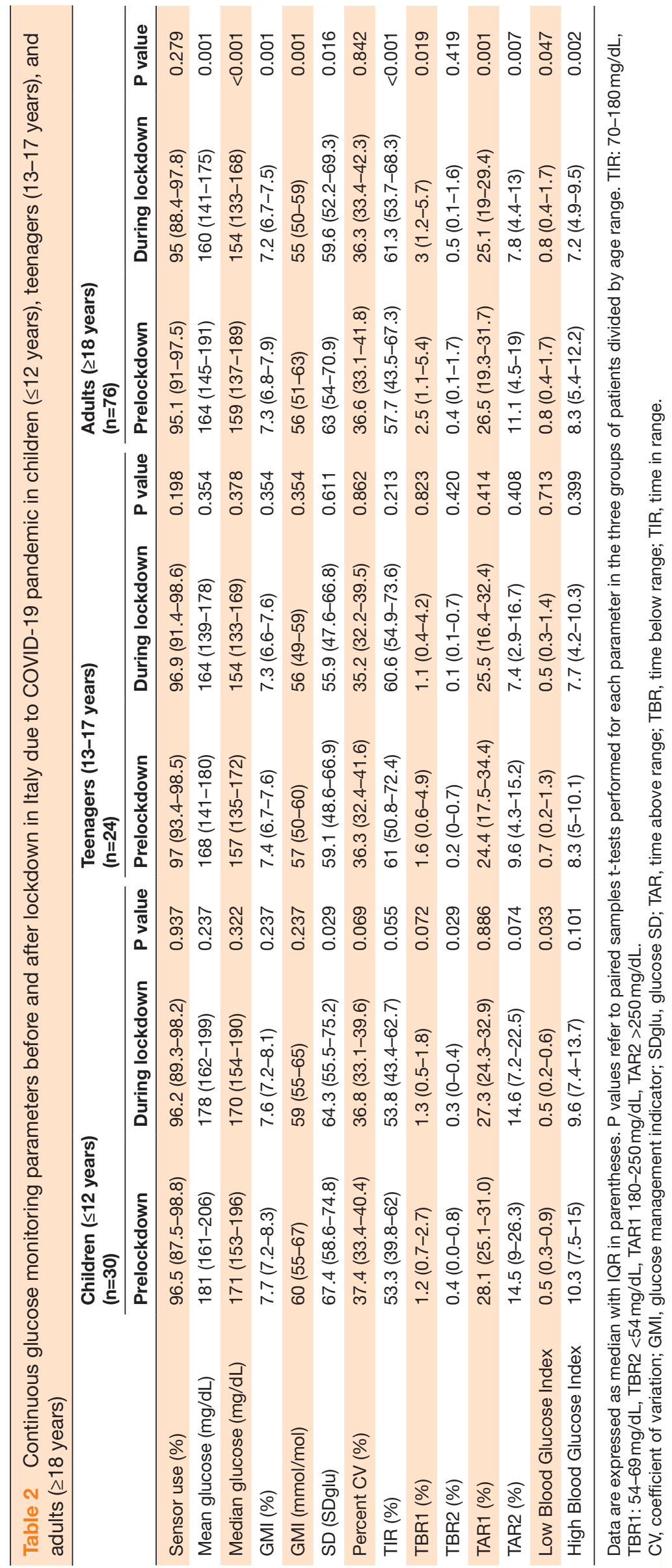


Table 3 Comparison among the three groups of adults identified by unsupervised clustering according to the difference in values of prelockdown and during lockdown of TIR $(\triangle \mathrm{TIR})$ and SD $(\triangle \mathrm{SDglu})$

\begin{tabular}{|c|c|c|c|c|}
\hline & $\begin{array}{l}\text { Improved TIR and } \\
\text { variability }(n=19)\end{array}$ & $\begin{array}{l}\text { Improved } \\
\text { variability }(n=38)\end{array}$ & $\begin{array}{l}\text { Worsened } \\
\text { variability }(n=19)\end{array}$ & $P$ value \\
\hline$\Delta \mathrm{TIR}(\%)$ & $32.9(18.5-39.0)^{*}$ & $3.6(-1.5-7.6)$ & $-5.0(-8.8-4.0)$ & $<0.001$ \\
\hline TIR prelockdown (\%) & $44.6(41.0-51.5)^{\star}$ & $59.4(50.5-69.2)$ & $66.6(42.7-73.2)$ & 0.002 \\
\hline TIR during lockdown (\%) & $60.1(56.6-64.7)$ & $60.7(53.0-72.5)$ & $62.7(41.9-69.6)$ & 0.768 \\
\hline SD prelockdown & $66.8(64.7-75.3)$ & $58.5(52.1-71.5)$ & $55.1(47.2-63.8) \ddagger$ & 0.043 \\
\hline Median glucose prelockdown (mg/dL) & $188(171-192)^{\star}$ & $156(137-171)$ & $148(129-190)$ & 0.016 \\
\hline Median glucose during lockdown (mg/dL) & $159(143-167)$ & $150(133-166)$ & $146(127-181)$ & 0.914 \\
\hline Age (years) & $56.8(28.9-60.9)$ & $35.3(26.4-48.7)^{\star}$ & $51.9(42.5-58.5)$ & 0.003 \\
\hline Female, n (\%) & $11(57.9)$ & $16(42.1)$ & $10(52.6)$ & 0.491 \\
\hline $\operatorname{BMI}\left(\mathrm{kg} / \mathrm{m}^{2}\right)$ & $25.8(22.3-27.8)$ & $24.7(21.6-26.2)$ & $24.5(22.4-26.8)$ & 0.592 \\
\hline Patients with severe hypoglycemia, $n(\%)$ & $1(5.3)$ & $0(0.0)$ & $0(0.0)$ & n.a. \\
\hline IPAQ: total physical activity/day, METs & $3158(1440-5700)^{*}$ & $1590(889-2895)$ & $700(420-870)$ & 0.009 \\
\hline IPAQ: high-intensity physical activity/day, METs & $1520(0-3780)$ & $480(0-1200)$ & $0(0-0)$ & 0.053 \\
\hline IPAQ: moderate physical activity/day, METs & $810(240-1680)$ & $480(60-840)$ & $440(15-900)$ & 0.981 \\
\hline IPAQ: walking/day, METs & $360(113-1260)$ & $385(98-810)$ & $250(28-563)$ & 0.143 \\
\hline IPAQ: mean sitting time/day (minutes) & $360(165-454)$ & $308(229-480)$ & $345(263-495)$ & 0.744 \\
\hline PSS: perceived stress & $16(10-21)$ & $11(8-18)$ & $18(13-25) \S$ & 0.020 \\
\hline \multicolumn{5}{|l|}{ Patients working during lockdown } \\
\hline No, n (\%) & $9(47.4)$ & $12(40.0)$ & $7(36.8)$ & 0.881 \\
\hline
\end{tabular}

Data are expressed as median with IQR in parentheses. Pairwise comparisons were made after Bonferroni adjustment.

${ }^{*} \mathrm{P}<0.05$ vs all other groups.

$\dagger \mathrm{P}<0.05$ for all pairwise comparisons.

$\ddagger P<0.05$ vs 'improved variability' group.

$\S \mathrm{P}<0.05 \mathrm{vs}$ 'improved TIR and variability' group.

BMI, body mass index; IPAQ, International Physical Activity Questionnaire-Short Form; MET, metabolic equivalent; n.a., not assessed; PSS, Perceived Stress Scale; SDglu, glucose SD; TIR, time in range.

group than the remaining two groups $(\mathrm{p}=0.009)$. Patients with worsened variability had significantly higher values at the PSS questionnaire than subjects belonging to the group improved variability $(\mathrm{p}=0.020)$.

\section{DISCUSSION}

Our study shows that during COVID-19 pandemic children and adults with T1D have had specific lockdowninduced effects on glucose levels, whereas teenagers with T1D did not show significant changes in glucose-related parameters, as measured by CGM. Additionally, we stratified adult patients according to their changes in TIR and glucose variability and identified potential factors contributing to modifications of those parameters. Finally, we were able to define a subgroup of adult patients who had worsened their glucose variability during lockdown.

The COVID-19 pandemic has made increasingly clear how people's routine activities, lifestyle, and access to clinical care may change in a rather fast and unpredictable way. The modifications imposed by the progressive lockdown strategy performed in Italy led to several consequences on the daily life of patients with T1D. Indeed, the first phase of lockdown, characterized by the suspension of the educational activities, and the second phase of complete lockdown, during which only essential services were running, have forced children and teenagers with T1D to a severe reduction of physical activity, a change in the time of meals and food choices.

By the analysis performed for the first time in children, we demonstrated that CGM metrics were mostly unchanged between the time period antecedent to the prelockdown, when the schools were still open, and the prelockdown phase, when the schools have been closed, except for a slight increase in TAR1. Additionally, we detected a significant improvement of glucose variability, as measured by SDglu, between prelockdown and during 
lockdown, mainly due to a reduction in TBR2. Those changes, which seems to be a specific consequence of lockdown, may reflect the relevant impact of the family environment, in which the mealtime was more regularly distributed during the day and the parents of patients with T1D might have payed closer attention to the management of T1D. However, it is difficult to establish whether those changes would have resulted in clinical benefits, considering that these patients were having a low rate of hypoglycemia before lockdown and thus further improvements would have been challenging.

In teenagers, CGM metrics were mostly comparable between the time period antecedent to the prelockdown (with schools opened) and the prelockdown (with school closed), except for a slight reduction in glucose variability (lower SDglu). Interestingly, no further changes were detected in the during the lockdown period. The data in teenagers led us to speculate that T1D management may be relatively independent of the heavy changes imposed by the lockdown. We can also propose that the influence of the family environment for T1D control may be less relevant in teenagers, differently from children, due to the progressive detachment from the family characterizing that period of life due to the desire for independence and autonomy.

Adult patients with T1D showed a significant improvement in the majority of the CGM metrics during lockdown period when compared with the prelockdown. Interestingly, no difference at all was detected between the 20 days antecedent to the prelockdown (corresponding to the schools-open period) and the 20 days immediately before lockdown, meaning that all changes in T1D management were strictly related to lockdown. Those data are in line with the results of a recent study, published while our article was still under writing. ${ }^{13}$ The study by Bonora et al investigated three time periods, 3 months before, 1 week before and 1 week after lockdown in Italy in 33 patients with T1D, showing a beneficial effect of lockdown on average glucose and SDglu, derived by CGM (FreeStyle Libre device). Additionally, most of the changes in CGM metrics were related to improvement in TIR and reduction of hyperglycemia (measured as TAR), whereas no difference in TBR was detected. The authors concluded that beneficial effects on glucose management in adults with T1D might be detected early after lockdown. ${ }^{13}$ Even though this study has relevant differences with the present study, including the number of subjects, the selection criteria of the patients, the outpatient setting, and the design of the study, our results perfectly fit, providing support to the hypothesis that T1D management may improve in early and intermediate periods after lockdown. The separate analysis in patients aged 18.0-25.9 years showed that young adult patients in this age range had a slightly different behavior in CGM metrics than older adults, supporting the differences highlighted in the T1D Exchange study. ${ }^{26}$ Additionally, our data are in line also with two more recent studies performed in 55 and 207 adult patients with type
1 diabetes in Italy. ${ }^{14}{ }^{15}$ Interestingly, the study by Capaldo et al showed an increased TIR during lockdown, together with a significant decrease of $\% \mathrm{CV}$ due to lower TBR and TAR, mostly in young adults ( $<35$ years old), men, and subjects using multiple daily insulin injections. ${ }^{15}$

Considering that the lockdown may have led to severely different conditions in adults with T1D, depending on working activities, physical activity, and potential consequences related to perceived stress, we attempted to further stratify adult patients according to their glucose management by selecting TIR as measurement of adequate glucose control and SDglu as a parameter of glucose variability. Furthermore, we reached all our patients by phone within 2 weeks after the study period to draw important information on the concomitant conditions that may have influenced the management of T1D, as data on physical activity and perceived stress with validated questionnaires. The clustering analysis allowed us to identify a group of patients who improved TIR and glucose variability (one-fourth of the patients) and two groups of patients who did not show significant changes in TIR but differed for SDglu, which was improved in half of the patients and worsened in the remaining onefourth of the subjects. The analysis of those three groups revealed a prevalent positive impact of higher levels of physical activity in the first group, whereas young age and lower glycated hemoglobin prelockdown were relevant characteristics of those with unchanged TIR and improved glucose variability. Notably, a higher perceived stress seemed to play a relevant role in the group of patients whose glucose variability worsened, even in the absence of changes in TIR between prelockdown and during lockdown. We can speculate that the perceived stress in this subgroup of patients might be related either to the general situation due to lockdown or to the knowledge of the potential increased risk of worse prognosis in case of COVID-19 infection. We proposed that the administration of PSS may be a simple tool to assess their psychological stress-related consequences due to lockdown, which may be helpful to select patients with T1D who may be at higher risk of having worsened glucose variability. Finally, we could not demonstrate an effect of working activity and type of employment, type of insulin treatment, and type of CGM device on glucose management in adult patients.

The main limitation of our study was that we enrolled a very well-selected cohort of patients with T1D, that is, those under CGM monitoring and with sensor use of $>70 \%$ during the study period. This is particularly true for the subgroup of teenagers whose mean glycated hemoglobin levels are better than those reported in the literature and consistent with those of motivated patients. ${ }^{25}$ Therefore, those results may not be extended to all patients with T1D, whose glucose changes during lockdown should be addressed in targeted studies. Additionally, we did not investigate the food choices with structured interview, and we did not consider the socioeconomic status of the patients, which may have a 
relevance in glucose control. Finally, it must be acknowledged that data derived from the Dexcom CGM system are obtained automatically every $5 \mathrm{~min}$, whereas those derived from the FreeStyle Libre system are obtained when the patient actively undergoes a scan. However, we believe that those differences did not affect significantly the results of the study after considering the number of scans per day of our patients wearing FreeStyle Libre. Nevertheless, both types of CGM are approved for insulin dosing are widely used and facilitate the monitoring of the TIR. ${ }^{18}$ Nonetheless, this is the first study providing data on glucose control in a wide spectrum of patients with T1D, ranging from children to adults. Moreover, we presented for the first time an analysis of potential lockdown-related factors that may have contributed to the changes in glucose-related parameters.

\section{CONCLUSION}

Lockdown-related effects on glucose management in patients with T1D are rapid and age-specific, being improved in children and mostly unchanged in teenagers. During the lockdown, the family environment and its pace of life would seem to be more attentive or more dedicated in controlling the disease. In adult patients, even though a significant improvement in most of the CGM metrics was detected in the whole population, one-fourth of the patients may show a worsened glucose variability related to increased perceived stress. Our data provide highly relevant information for selection of patients with T1D that may be at higher risk of worsening their glucose management during lockdown.

Correction notice This article has been corrected since it was published. A column in table 3 was missing.

Acknowledgements We thank all patients with type 1 diabetes and their relatives for the immediate adhesion to our study.

Contributors Study design: GDD, UP and SZ. Data collection: GDD, GM, CB, LT, VDN, SM and GL. Data analysis: GDD and CB. Data interpretation: GDD, GM, AP, SZ and UP. Writing of the manuscript and final review: all authors.

Funding The authors have not declared a specific grant for this research from any funding agency in the public, commercial or not-for-profit sectors.

Competing interests None declared.

Patient consent for publication Not required.

Ethics approval The study was approved by the local ethics committee of the Policlinic (CE-AVEC, number 673/2020/0ss/AOUBo). Written informed consent was collected for data analysis in anonymous form.

Provenance and peer review Not commissioned; externally peer reviewed.

Data availability statement Data are available upon reasonable request.

Supplemental material This content has been supplied by the author(s). It has not been vetted by BMJ Publishing Group Limited (BMJ) and may not have been peer-reviewed. Any opinions or recommendations discussed are solely those of the author(s) and are not endorsed by BMJ. BMJ disclaims all liability and responsibility arising from any reliance placed on the content. Where the content includes any translated material, BMJ does not warrant the accuracy and reliability of the translations (including but not limited to local regulations, clinical guidelines, terminology, drug names and drug dosages), and is not responsible for any error and/or omissions arising from translation and adaptation or otherwise.

Open access This is an open access article distributed in accordance with the Creative Commons Attribution Non Commercial (CC BY-NC 4.0) license, which permits others to distribute, remix, adapt, build upon this work non-commercially, and license their derivative works on different terms, provided the original work is properly cited, appropriate credit is given, any changes made indicated, and the use is non-commercial. See: http://creativecommons.org/licenses/by-nc/4.0/.

ORCID IDs

Guido Di Dalmazi http://orcid.org/0000-0002-1291-8906

Claudio Bongiorno http://orcid.org/0000-0002-9092-9758

\section{REFERENCES}

1 Zhu N, Zhang D, Wang W, et al. A novel coronavirus from patients with pneumonia in China, 2019. N Engl J Med 2020;382:727-33.

2 John Hopkins University \& Medicine. Coronavirus resource center. Available: https://coronavirus.jhu.edu/map.html

3 Gazzetta Ufficiale. II PRESIDENTE del CONSIGLIO dei MINISTRI. Available: https://www.gazzettaufficiale.it/eli/id/2020/03/11/ 20A01605/sg

4 Richardson S, Hirsch JS, Narasimhan M, et al. Presenting characteristics, comorbidities, and outcomes among 5700 patients hospitalized with COVID-19 in the new York City area. JAMA 2020;323:2052

5 Guo L, Wei D, Zhang X, et al. Clinical features predicting mortality risk in patients with viral pneumonia: the MuLBSTA score. Front Microbiol 2019;10:2752.

6 Wu C, Chen X, Cai Y, et al. Risk factors associated with acute respiratory distress syndrome and death in patients with coronavirus disease 2019 pneumonia in Wuhan, China. JAMA Intern Med 2020;180:e200994.

7 Wang D, Hu B, Hu C, et al. Clinical characteristics of 138 hospitalized patients with 2019 novel coronavirus-infected pneumonia in Wuhan, China. JAMA 2020;323:1061-9.

8 Lind M, Polonsky W, Hirsch IB, et al. Continuous glucose monitoring vs conventional therapy for glycemic control in adults with type 1 diabetes treated with multiple daily insulin injections: the gold randomized clinical trial. JAMA 2017;317:379-87.

9 Beck RW, Riddlesworth T, Ruedy K, et al. Effect of continuous glucose monitoring on glycemic control in adults with type 1 diabetes using insulin injections: the diamond randomized clinical trial. JAMA 2017;317:371-8.

10 Heinemann L, Freckmann G, Ehrmann D, et al. Real-Time continuous glucose monitoring in adults with type 1 diabetes and impaired hypoglycaemia awareness or severe hypoglycaemia treated with multiple daily insulin injections (HypoDE): a multicentre, randomised controlled trial. Lancet 2018;391:1367-77.

11 Bolinder J, Antuna R, Geelhoed-Duijvestijn P, et al. Novel glucosesensing technology and hypoglycaemia in type 1 diabetes: a multicentre, non-masked, randomised controlled trial. Lancet 2016;388:2254-63.

12 Cherubini V, Bonfanti R, Casertano A, et al. Time in range in children with type 1 diabetes using treatment strategies based on nonautomated insulin delivery systems in the real world. Diabetes Technol Ther 2020;22:509-15.

13 Bonora BM, Boscari F, Avogaro A, et al. Glycaemic control among people with type 1 diabetes during Lockdown for the SARS-CoV-2 outbreak in Italy. Diabetes Ther 2020:1-11.

14 Maddaloni E, Coraggio L, Pieralice S, et al. Effects of COVID-19 Lockdown on glucose control: continuous glucose monitoring data from people with diabetes on intensive insulin therapy. Diabetes Care 2020;43:e86-7.

15 Capaldo B, Annuzzi G, Creanza A, et al. Blood glucose control during Lockdown for COVID-19: CGM metrics in Italian adults with type 1 diabetes. Diabetes Care 2020;43:e88-9.

16 Bergenstal RM, Beck RW, Close KL, et al. Glucose management indicator (GMI): a new term for estimating $\mathrm{A} 1 \mathrm{c}$ from continuous glucose monitoring. Diabetes Care 2018;41:2275-80.

17 Rodbard D. Display of glucose distributions by date, time of day, and day of week: new and improved methods. J Diabetes Sci Technol 2009;3:1388-94.

18 Danne T, Nimri R, Battelino T, et al. International consensus on use of continuous glucose monitoring. Diabetes Care 2017;40:1631-40.

19 Battelino T, Danne T, Bergenstal RM, et al. Clinical targets for continuous glucose monitoring data interpretation: recommendations from the International consensus on time in range. Diabetes Care 2019;42:1593-603.

20 Kovatchev BP, Straume M, Cox DJ, et al. Risk analysis of blood glucose data: a quantitative approach to optimizing the control of insulin dependent diabetes. J Theor Med 2000;3:1-10.

21 Clarke W, Kovatchev B. Statistical tools to analyze continuous glucose monitor data. Diabetes Technol Ther 2009;11:45-54. 
22 Cacciari E, Milani S, Balsamo A, et al. Italian cross-sectional growth charts for height, weight and BMI (2 to $20 \mathrm{yr}$ ). J Endocrinol Invest 2006;29:581-93.

23 Craig CL, Marshall AL, Sjöström M, et al. International physical activity questionnaire: 12 -country reliability and validity. Med Sci Sports Exerc 2003;35:1381-95.

24 Mondo M, Sechi C, Cabras C. Psychometric evaluation of three versions of the Italian perceived stress scale. Curr Psychol 2019;52:1.
25 Hermann JM, Miller KM, Hofer SE, et al. The Transatlantic $\mathrm{HbA}_{1}$ gap: differences in glycaemic control across the lifespan between people included in the US T1D Exchange Registry and those included in the German/Austrian DPV registry. Diabet Med 2020;37:848-55

26 Foster NC, Beck RW, Miller KM, et al. State of Type 1 Diabetes Management and Outcomes from the T1D Exchange in 2016-2018 [published correction appears in Diabetes Technol Ther. 2019 Apr;21(4):230].. Diabetes Technol Ther 2019;21:66-72. 